in vivo $35: 23-30(2021)$

doi:10.21873/invivo.12228

Review

\title{
Sensitivity, Specificity and the Diagnostic Accuracy of PET/CT for Axillary Staging in Patients With Stage I-III Cancer: A Systematic Review of The Literature
}

\author{
JUDI KASEM $^{1}$, UMAR WAZIR ${ }^{1,2}$ and KEFAH MOKBEL ${ }^{1}$ \\ ${ }^{1}$ London Breast Institute, Princess Grace Hospital, London, U.K.; \\ ${ }^{2}$ Department of General Surgery, Khyber Teaching Hospital, Peshawar, Pakistan
}

\begin{abstract}
Background/Aim: Axillary lymph node (ALN) status plays a key role in the staging of breast cancer. Positron Emission Tomography/Computed Tomography (PET/CT) using 18-Fluorodeoxyglucose ( $\left.{ }^{18} \mathrm{FDG}\right)$ can visualise ALN metastasis. However, its utility compared to current methods is unclear. We systematically reviewed the role of ${ }^{18}$ FDG PET/CT in breast cancer staging. Materials and Methods: PubMed, Ovid and Cochrane were searched systematically up until August 2020. Included papers had true positive (TP), false positive (FP), true negative (TN) and false negative (FN) rates, sensitivity, specificity, accuracy, positive $(P P V)$ and negative predictive value (NPV). Results: Nine studies $(n=1486)$ were included, showing: i) sensitivity $=52.2 \%$, ii) specificity $=91.6 \%$, iii) $P P V=77.8 \%$, iv) $N P V=77.2$, and v) accuracy $=77.3 \%$. Conclusion: ${ }^{18} F D G-$ PET/CT has a low sensitivity but high specificity for ALN disease. Therefore, ultrasound-guided biopsy could be considered in a positive CT/PET. Modest accuracy prohibits the use of ${ }^{18} \mathrm{FDG}-\mathrm{PET} / \mathrm{CT}$ alone in axillary staging. Prospective research using standardised protocols and quantitative cut-off points is warranted.
\end{abstract}

Axillary lymph node (ALN) assessment plays a key role in the staging and upstaging of breast cancer. It is an important predictor of the survival and disease recurrence in patients

This article is freely accessible online.

Correspondence to: Kefah Mokbel, London Breast Institute, the Princess Grace Hospital, 45 Nottingham Place, London W1U 5NY, United Kingdom. Tel: +44 2079082040, e-mail: kefah.mokbel@hotmail.com

Key Words: Breast cancer, CT/PET, imaging, sentinel node biopsy, axillary disease.
(1), thus determining prognosis and treatment (2). Axillary staging can also be used for therapeutic response monitoring (3). Current guidelines suggest sentinel lymph node biopsy (SNB) as the preferred technique for axillary staging, followed by axillary lymph node dissection (ALND) in patients with positive SNB results (4). SNB is not without surgical risk, and thus, a non-invasive alternative is highly desirable $(3,5)$.

Positron Emission Tomography (PET) using 18Fluorodeoxyglucose $\left({ }^{18} \mathrm{FDG}\right)$ highlights metabolically active locations in the body. When combined with Computed Tomography (CT) as ${ }^{18} \mathrm{FDG}-\mathrm{PET} / \mathrm{CT}$, this imaging modality could increase diagnostic accuracy significantly (6). A PET/CT scan could be assessed by one of two methods: one method being a qualitative, visual assessment and the other, by calculating the maximum standardized uptake value (SUVmax), which is a semi-quantitative method (7). When using visual assessment alone, experienced personnel provide a rating of suspected tumours, such as using a scale of " 0 for normal, 1 for equivocal, 2 for probably abnormal and 3 for abnormal detecting subclinical nodal metastasis" (8) or simply deciding on whether uptake was higher than background activity (9).

SUVmax is calculated by dividing maximum activity in a region of interest $(\mathrm{MBq} / \mathrm{mL})$ by injected dose $(\mathrm{MBq})$ divided by body weight (g) (10). SUVmax cut-off ranges across different studies from 0.8 to $3(1,2,8)$. SUVmax can serve as an adjunct for reference when using visual assessment $(1,2,11)$.

$\mathrm{PET} / \mathrm{CT}$ is not only useful for the detection of locoregional lymph node involvement but also distant metastases (12). It has been suggested as potentially replacing other methods for detecting distant metastatic involvement (12), however when assessing axillary lymph node involvement, low sensitivity and high specificity have been reported (13). PET/CT has a reasonable overall accuracy with high specificity, hence it can be used for 
selecting patients for either SNB or ALND (10), thereby avoiding unnecessary procedures (14). In addition, most studies have only focused on a small number of tumour types, especially invasive ductal carcinoma (7), therefore more research is required to assemble the findings of the current literature on this topic.

Current literature is limited due to variations in study design, variety in breast cancer subtypes included, as well as methods of determining malignancy in scans (13). PET/CT is comparatively less sensitive in detecting ALN status when the primary tumour has a low uptake of ${ }^{18}$ FDG (7). Similar issues have been reported for small lesions (12) due to its restricted spatial resolution (3). False-negative $(\mathrm{FN})$ results are associated with a higher $\mathrm{T}$ stage (15). An increased likelihood of FNs can be influenced by i) increased age, ii) positive oestrogen receptor status and iii) mixed tissue histology, whereas being HER-2 positive can decrease the likelihood (16).

There is currently no consensus on the role of PET/CT in guiding therapy. Whilst a preponderance of authors do not believe it to be a substitute for SNB or ALND (2) with no role in the management of early breast cancer patients (17), some believe that it could determine whether SNB or ALND is required $(10,14)$ and potentially screen out patients with non-suspicious nodes (18).

We aim to systematically review the literature regarding the diagnostic performance of ${ }^{18} \mathrm{FDG}$ PET/CT in the assessment of lymphatic metastasis in stage I-III breast cancer, and discuss the potential role this modality could have in the management of breast cancer.

\section{Materials and Methods}

Data search. PubMed, Ovid and Cochrane library databases were searched to identify any relevant publications. The PubMed search was conducted on 29th July 2020. The Ovid database was searched on the 29th July 2020. This search included the Embase, Emcare and Ovid MEDLIE(R) and Epub Ahead of Print, In-Process \& Other Non-Indexed Citations, Daily and Versions (R). The search terms used were as follows: "PET OR positron emission tomography" AND "CT or computerized tomography OR computerised tomography" AND "axillary" AND "staging".

The Cochrane library search was conducted on 15th August 2020 using the terms "PET/CT" and "axilla" including any associated terms.

Inclusion \& exclusion criteria. Both prospective and retrospective studies were included. Papers were included if they assessed the sensitivity, specificity and diagnostic accuracy of PET/CT for axillary lymph node metastases. Only studies with full text articles published in English were included. In addition, studies were included if they focused on either visual assessment or using SUVmax of PET/CT scans. Finally, the participant cohort had to be limited to patients with Stage I-III cancer.

Studies were excluded if they did not specify their standard of reference or method of evaluation of PET/CT scans. Papers were also excluded if they did not compare PET/CT to histopathological findings. Studies with sample sizes of below 50 were excluded. Papers were excluded if true positive (TP), false positive (FP), true negative (TN) or false negative (FN) rates could not be extracted to form a 2 by 2 contingency table. Papers were excluded where participants had a PET/CT after or during neo-adjunctive therapy. Other exclusion criteria involved Stage IV breast cancer patients.

Data extraction and management. Papers were reviewed and information including authors, publication date, time, location and study type, was collected. Participants' average age was extracted, along with breast cancer subtype. Standard of reference and information on ${ }^{18} \mathrm{FDG}$ dose were also included. All papers had true positive (TP), false positive (FP), true negative (TN) and false negative (FN) rates excerpted, along with sensitivity, specificity, positive predictive value (PPV), negative predictive value (NPV) and diagnostic accuracy. This extracted data were combined to calculate overall pooled means for sensitivity, specificity, PPV, NPV and accuracy for papers using visual assessment. Pooled values were calculated by combining the overall results for TP, FP, TN, and FN from each respective study.

A pooled analysis could not be carried out for the SUVmax method as studies used different SUVmax cut offs.

Quality analysis. The quality of the studies included in this review was assessed using Section A and B of the CASP critical appraisal tool for diagnostic studies.

\section{Results}

Overall, 1,106 articles (PubMed: 255, Ovid: 830, Cochrane: 21) were identified and two further articles were found through assessing article bibliographies. Following removal of duplicates 892 remained. After screening of abstracts, 117 full text articles were assessed, 37 full texts were not available, shorting the assessment to 80 articles. Studies were excluded due to not focussing on either axillary lymph node metastases $(n=5)$ or evaluation of diagnostic accuracy $(n=24)$. One article was excluded for using a different tracer (not ${ }^{18} \mathrm{FDG}$ ). Other excluded studies did not have primary data and were general reviews of the use of PET/CT in axillary staging $(n=9)$.

Finally, ten studies were selected for this systematic review (Figure 1) (Table I). Eight studies (2, 7, 9, 16, 19-22) were retrospective and two were prospective $(1,8)$. Seven papers used both visual assessment as well as SUVmax (1, $7-9,16,20,22)$, whereas one used SUVmax as a concomitant only indicator $(21,23)$. Two studies used only SUVmax (2) or visual (19) assessment, while all studies used histopathology assessment as the standard of reference. Most studies included patients with ductal or lobular carcinomas $(1,2,9,19,20)$, and one study did not define what other cancer subtypes were included (8). Another paper did not specify what breast cancer subtype was included (22). Doses of ${ }^{18} \mathrm{FDG}$ differed across the papers but the most common one was $3.7 \mathrm{Mbq} / \mathrm{kg}(1,7,9)$. Overall, 1,484 patients underwent PET/CT with visual assessment being the main criterion for ALN assessment, whereas 373 underwent SUVmax as the main criterion. 


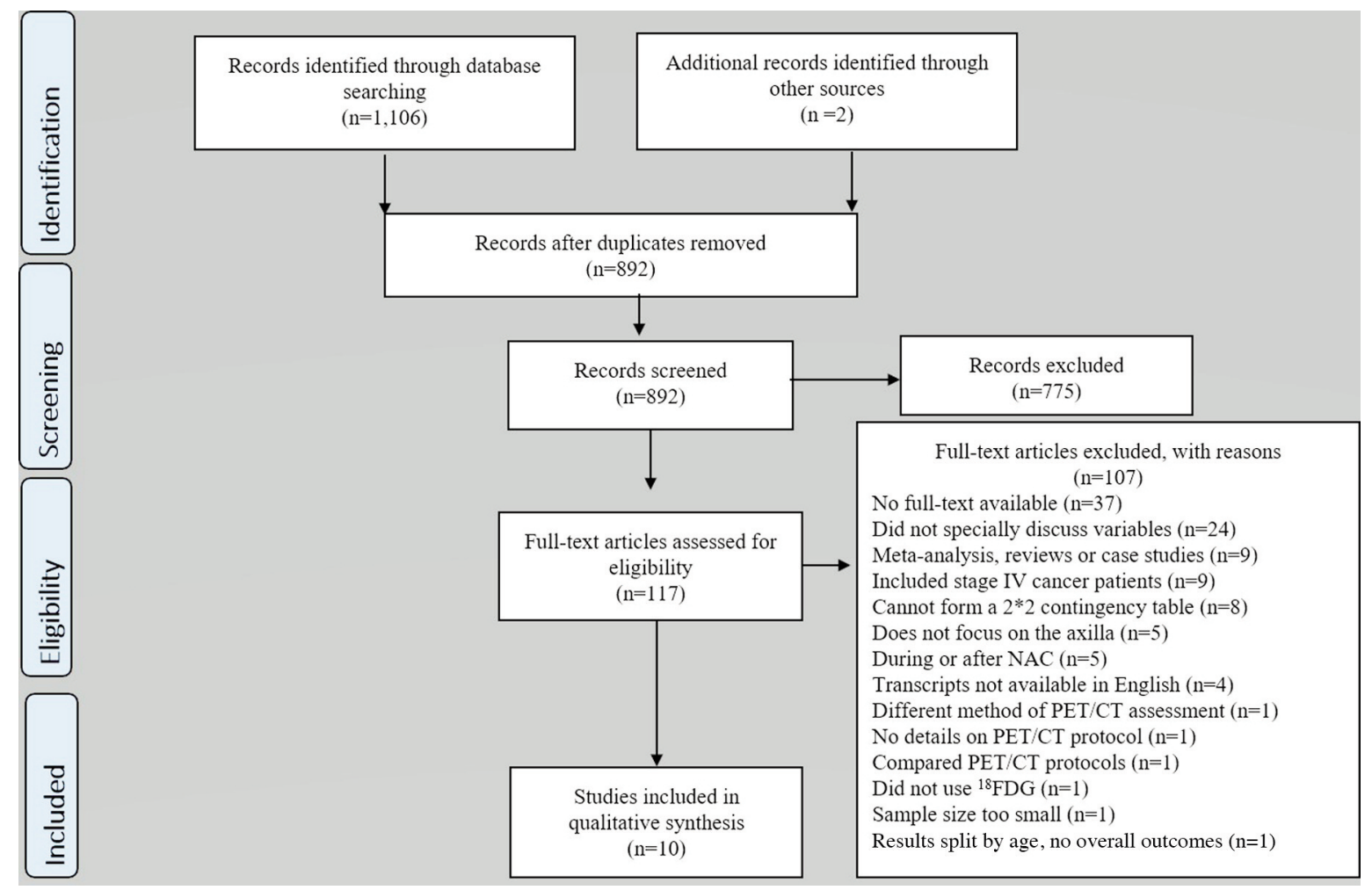

Figure 1. A PRISMA flowchart of data collected and selected studies.

Quality assessment. The critical analysis of the studies is outlined in Table II. Some studies only included tumour and node staging rather than overall staging $(8,9,16,19,21,22)$. These were concluded to be Stage I-III as no mention of peripheral metastasis occurred.

Diagnostic accuracy. Most papers only reported diagnostic accuracy for visual assessment $(8,9,16,19-21,24)$ (Table III), whereas only three papers offered diagnostic accuracy on the SUVmax method $(1,2,22)$. The sensitivity for visual analysis ranged from $18.5-85 \%(9,22)$, whereas specificity ranged from $77.9-97.1 \%(9,16)$. Overall specificity was high, with seven studies finding it above $90 \%$ (7-9, 12, 19, $21,22)$. PPV was generally above $70 \%(1,8,16,19,22)$, however, Jeong et al. (20) have found it to be $37 \%$. Overall accuracy was above $70 \%$, with the exception of Jeong et al., who reported an accuracy of $69.1 \%$ (20).

Regarding SUVmax (Table IV), when the cut off was set at 1.1 for Mori et al. (21), overall accuracy was $94 \%$ whereas Ueda et al. (1) have found that even when SUVmax was set at different cut off points, overall best accuracy was at a cut off of 0.8 , with a diagnostic accuracy of $80.9 \%$.

When assessing for the pooled analysis for papers using visual qualitive assessment as the PET/CT criteria, 1,486 axillae were included in the analysis. PET/CT correctly identified malignancy in 281 axillae, however, it missed malignancy in 257 of them. Hence, pooled sensitivity was $52.2 \%$. Pooled specificity was $91.6 \%$, as PET/CT correctly declared 868 axillae as clear of lymph node metastasis. Overall, pooled diagnostic accuracy was $77.3 \%$.

\section{Discussion}

This study systematically reviewed the diagnostic performance of ${ }^{18} \mathrm{FDG}$ PET/CT in the assessment of ALN metastasis in patients with stage I-III breast cancer. Overall, across the papers included in this review, many studies found similar findings for sensitivity and specificity. PET/CT was shown to have high specificity but low sensitivity, leading to a moderate overall accuracy (10).

Our calculated pooled sensitivity of $52 \%$ is similar to that reported in a meta-analysis performed by Zhang et al. [56\%; $95 \%$ confidence interval $(\mathrm{CI})=47 \%-63 \%](13)$. The sensitivity in our pooled analysis ranged from $18.5 \%$ to $85 \%(9,22)$. The likelihood of FNs can be influenced by old age, primary tumour characteristics and volume of axillary disease (16).

Mori et al. (22) did not state the tissue histology of the cancer included in their study, which could have affected their sensitivity. However, they did also use Time-Of-Flight ${ }^{18}$ FDG PET/CT, which performs better than normal PET/CT (25). 
Table I. A summary table of papers included in this review.

\begin{tabular}{|c|c|c|c|c|c|c|c|c|}
\hline Author & Type & $\begin{array}{l}\text { Region and } \\
\text { time period }\end{array}$ & $\begin{array}{l}\text { Number } \\
\text { of cases }\end{array}$ & $\begin{array}{c}\text { Average } \\
\text { age (years) }\end{array}$ & $\begin{array}{l}\text { Subtype of } \\
\text { carcinoma }\end{array}$ & $\begin{array}{l}\text { Standard of } \\
\text { reference }\end{array}$ & $\begin{array}{c}\text { Dose of } \\
\text { FDG }\end{array}$ & $\begin{array}{l}\text { Method of } \\
\text { evaluation }\end{array}$ \\
\hline $\begin{array}{l}\text { Chae et al. } \\
2008 \text { (2) }\end{array}$ & Retrospective & $\begin{array}{c}\text { Korea } \\
\text { Mar-Nov } \\
2003\end{array}$ & 108 & $\begin{array}{c}\text { Mean }=48.6 \\
\text { Range }=27-75\end{array}$ & $\begin{array}{l}\text { Ductal, Lobular, } \\
\text { Mucinous, Medullary }\end{array}$ & HP & $\begin{array}{c}\text { Five } \\
\text { millicuries } \\
\text { of FDG } \\
(185 \mathrm{MBq})\end{array}$ & SUVmax \\
\hline $\begin{array}{l}\text { Heusner et al. } \\
2009 \text { (19) }\end{array}$ & Retrospective & $\begin{array}{l}\text { Germany } \\
\text { Sept 2007- } \\
\text { Dec } 2008\end{array}$ & 61 & $\begin{array}{l}\text { Mean }=56 \pm 13 \\
\text { Range }=28-78\end{array}$ & $\begin{array}{c}\text { Ductal, Lobular, } \\
\text { Mixed ductal/lobular, } \\
\text { Undifferentiated, } \\
\text { Mucinous, } \\
\text { Neuroendocrine, } \\
\text { Necrotic, Tubular }\end{array}$ & HP & $\begin{array}{c}271 \pm 35 \mathrm{MBq} \\
\text { FDG } \\
\text { Range }= \\
210-360 \mathrm{MBq}\end{array}$ & Visual \\
\hline $\begin{array}{l}\text { Jeong et al. } \\
2014 \text { (20) }\end{array}$ & Retrospective & $\begin{array}{l}\text { South Korea } \\
\text { Jan 2010- } \\
\text { Sept } 2013\end{array}$ & 178 & $\begin{array}{c}\text { Mean }=54.9 \pm 9.8 \\
\text { Range }=33-82\end{array}$ & $\begin{array}{l}\text { Ductal, Lobular, } \\
\text { DCIS other }\end{array}$ & HP & $5.2 \mathrm{MBq} / \mathrm{kg}$ & $\begin{array}{l}\text { Visual \& } \\
\text { SUVmax }\end{array}$ \\
\hline $\begin{array}{l}\text { Kong et al. } \\
2010(21)\end{array}$ & Retrospective & $\begin{array}{c}\text { Korea } \\
\text { May 2007- } \\
\text { June } 2009\end{array}$ & 143 & Mean $=50.1 \pm 9.6$ & $\begin{array}{c}\text { Ductal, Lobular, } \\
\text { Tubular, } \\
\text { Micropapillary, } \\
\text { Metaplastic, Medullary, } \\
\text { Mucinous, Cribriform, } \\
\text { Microinvasive, Apocrine }\end{array}$ & HP & $370 \mathrm{MBq}$ & $\begin{array}{c}\text { Visual \& } \\
\text { SUVmax } \\
\text { served as } \\
\text { concomitant } \\
\text { only }\end{array}$ \\
\hline $\begin{array}{l}\text { Kutlutürk } \\
\text { et al. } 2019 \\
\text { (15) }\end{array}$ & Retrospective & $\begin{array}{l}\text { Turkey } \\
\text { Jan 2013- } \\
\text { Sept 2017 }\end{array}$ & 232 & $\begin{array}{c}\text { Mean }= \\
50.65 \pm 12.35 \\
\text { Range }=25-85\end{array}$ & Invasive carcinoma & HP & $0.1 \mathrm{mg} / \mathrm{kg}$ & $\begin{array}{l}\text { Visual \& } \\
\text { SUVmax }\end{array}$ \\
\hline $\begin{array}{l}\text { Machida et al. } \\
2019 \text { (9) }\end{array}$ & Retrospective & $\begin{array}{c}\text { Japan } \\
\text { Dec 2005- } \\
\text { Nov } 2009\end{array}$ & 227 & $\begin{array}{c}\text { Median }=55 \\
\text { Range }=26-87\end{array}$ & $\begin{array}{c}\text { DCIS, Ductal, } \\
\text { Lobular, Mucinous, } \\
\text { Medullary, } \\
\text { Metaplastic, Apocrine }\end{array}$ & $\mathrm{HP}$ & $3.7 \mathrm{MBq} / \mathrm{kh}$ & $\begin{array}{l}\text { Visual \& } \\
\text { SUVmax }\end{array}$ \\
\hline $\begin{array}{l}\text { Mori et al. } \\
2019 \text { (22) }\end{array}$ & Retrospective & $\begin{array}{c}\text { Japan } \\
\text { Jan 2016- } \\
\text { June } 2018\end{array}$ & 82 & $\begin{array}{c}\text { Mean }=59.3 \\
\text { Range }=30-84\end{array}$ & Not mentioned & HP & $\begin{array}{c}3.7 \mathrm{MBq} / \mathrm{kg} \\
0.1 \mathrm{mCi} / \mathrm{kg}\end{array}$ & $\begin{array}{l}\text { Visual \& } \\
\text { SUVmax }\end{array}$ \\
\hline $\begin{array}{l}\text { Park et al. } \\
2017 \text { (7) }\end{array}$ & Retrospective & $\begin{array}{c}\text { Korea } \\
\text { Jan 2009- } \\
\text { Mar 2015 }\end{array}$ & $\begin{array}{l}142(3 \mathrm{had} \\
\text { bilateral } \\
\text { tumours, } \\
\text { total } 144 \\
\text { lesions) }\end{array}$ & $\begin{array}{l}\text { Median }=49.0 \\
\text { Range }=28-84\end{array}$ & $\begin{array}{c}\text { Lobular, Mucinous, } \\
\text { Tubular }\end{array}$ & HP & $\begin{array}{l}3.7-5.5 \\
\mathrm{MBq} / \mathrm{kg}\end{array}$ & $\begin{array}{l}\text { Visual \& } \\
\text { SUVmax }\end{array}$ \\
\hline $\begin{array}{l}\text { Ueda et al. } \\
2008 \text { (1) }\end{array}$ & Prospective & $\begin{array}{c}\text { Japan } \\
\text { April 2005- } \\
\text { August } 2007 .\end{array}$ & 183 & $\begin{array}{c}\text { Mean }=57 \\
\text { Range }=32-81\end{array}$ & $\begin{array}{c}\text { DCIS, Ductal, } \\
\text { Lobular, Apocrine, } \\
\text { Mucinous, } \\
\text { Squamous, Paget }\end{array}$ & HP & $3.7 \mathrm{Mbq} / \mathrm{kg}$ & $\begin{array}{l}\text { Visual \& } \\
\text { SUVmax }\end{array}$ \\
\hline $\begin{array}{l}\text { Veronesi et al. } \\
2007 \text { (8) }\end{array}$ & Prospective & $\begin{array}{c}\text { Italy } \\
\text { July } 1996- \\
\text { July } 2000\end{array}$ & 236 & $\begin{array}{c}\text { Median }=49 \\
\text { Range }=24-79\end{array}$ & $\begin{array}{l}\text { Ductal, Lobular, } \\
\text { Other invasive }\end{array}$ & HP & $5.3 \mathrm{Mbq} / \mathrm{kg}$ & $\begin{array}{l}\text { Visual \& } \\
\text { SUVmax }\end{array}$ \\
\hline
\end{tabular}

HP: Histopathology.

Jeong et al. (20) have found sensitivity to be $20.8 \%$, however, the study participants had tumours that were in their early stage and micrometastatic nodes. As PET/CT has a small spatial resolution, small ALN metastasis can be left undetected $(3,16,20,26-29)$ leading to an increased falsenegative rate and a decreased sensitivity. This especially occurs for micrometastases as observed by Kutlutürk et al. (16), who have found that these accounted for $32 \%$ of FNs, as ${ }^{18}$ FDG uptake depends on the nature of the tumour (9). A review of the recent literature suggests that such deposits may not warrant ALND, and could be treated using adjuvant chemotherapy and endocrine therapy (30).

We have observed a relatively high specificity of $91.6 \%$ with a range from $77.9 \%$ to $97.1 \%(9,16)$. This finding is consistent with the prior meta-analysis by Zhang et al. (13), who have reported an overall specificity of $91 \%$ (95\% CI: $87 \%-93 \%$ ) for visual analysis. Wahl et al. (28) have found in a prospective multicentre study that FN findings occur in patients with reduced tumour burdens (28). Due to its relatively high specificity, PET/CT could be used to avoid 
Kasem et al: Diagnostic Accuracy of PET/CT for Axillary Staging in Breast Cancer

Table II. A CASP critical analysis of the papers included in this review.

\begin{tabular}{|c|c|c|c|c|c|c|c|c|c|c|}
\hline $\begin{array}{l}\text { CASP } \\
\text { Criteria }\end{array}$ & $\begin{array}{c}\text { Chae } \\
\text { et al. }(2)\end{array}$ & $\begin{array}{l}\text { Heusner } \\
\text { et al. }(19)\end{array}$ & $\begin{array}{l}\text { Jeong } \\
\text { et al. }(20)\end{array}$ & $\begin{array}{c}\text { Kong } \\
\text { et al. }(21)\end{array}$ & $\begin{array}{l}\text { Kutlutürk } \\
\text { et al. (15) }\end{array}$ & $\begin{array}{l}\text { Machida } \\
\text { et al. }(9)\end{array}$ & $\begin{array}{c}\text { Mori } \\
\text { et al. }(22)\end{array}$ & $\begin{array}{c}\text { Park et al. } \\
2017 \text { (7) }\end{array}$ & $\begin{array}{l}\text { Ueda } \\
\text { et al. }(1)\end{array}$ & $\begin{array}{l}\text { Veronesi } \\
\text { et al. }(8)\end{array}$ \\
\hline $\begin{array}{l}\text { Clearly focused } \\
\text { question }\end{array}$ & $\mathrm{Y}$ & $\mathrm{Y}$ & $\mathrm{Y}$ & Y & $\mathrm{Y}$ & $\mathrm{Y}$ & Y & $\mathrm{Y}$ & $\mathrm{Y}$ & Y \\
\hline $\begin{array}{l}\text { Appropriate } \\
\text { reference standard }\end{array}$ & $\mathrm{Y}$ & $\mathrm{Y}$ & $\mathrm{Y}$ & Y & $\mathrm{Y}$ & $\mathrm{Y}$ & Y & Y & $\mathrm{Y}$ & $\mathrm{Y}$ \\
\hline $\begin{array}{l}\text { All patients received } \\
\text { diagnostic test and } \\
\text { reference standard }\end{array}$ & $\mathrm{Y}$ & $\mathrm{Y}$ & Y & Y & $\mathrm{Y}$ & $\mathrm{Y}$ & Y & Y & $\mathrm{Y}$ & Y \\
\hline $\begin{array}{l}\text { Results were not } \\
\text { influenced by the } \\
\text { reference standard }\end{array}$ & $\mathrm{Y}$ & $\mathrm{Y}$ & $\mathrm{Y}$ & $\mathrm{Y}$ & $\mathrm{Y}$ & $\mathrm{Y}$ & $\mathrm{Y}$ & $\mathrm{Y}$ & $\mathrm{Y}$ & $\mathrm{Y}$ \\
\hline $\begin{array}{l}\text { Disease status } \\
\text { clearly described }\end{array}$ & $\mathrm{Y}$ & - & $\mathrm{Y}$ & - & - & - & - & $\mathrm{Y}$ & $\mathrm{Y}$ & - \\
\hline $\begin{array}{l}\text { Methods provided with } \\
\text { sufficient detail }\end{array}$ & - & $\mathrm{Y}$ & $\mathrm{Y}$ & Y & - & $\mathrm{Y}$ & Y & - & $\mathrm{Y}$ & Y \\
\hline Results provided & $\begin{array}{c}\text { TP, FP, } \\
\text { TN, FN, } \\
\text { sensitivity, } \\
\text { specificity, } \\
\text { diagnostic } \\
\text { accuracy }\end{array}$ & $\begin{array}{c}\text { TP, FP, } \\
\text { TN, FN, } \\
\text { sensitivity, } \\
\text { specificity, } \\
\text { PPV, NPV, } \\
\text { diagnostic } \\
\text { accuracy }\end{array}$ & $\begin{array}{c}\text { TP, FP, } \\
\text { TN, FN, } \\
\text { sensitivity, } \\
\text { specificity, } \\
\text { PPV, NPV, } \\
\text { diagnostic } \\
\text { accuracy }\end{array}$ & $\begin{array}{c}\text { TP, FP, } \\
\text { TN, FN, } \\
\text { sensitivity, } \\
\text { specificity, } \\
\text { PPV, NPV }\end{array}$ & $\begin{array}{c}\text { TP, FP, } \\
\text { TN, FN, } \\
\text { sensitivity, } \\
\text { specificity, } \\
\text { PPV, NPV, } \\
\text { diagnostic } \\
\text { accuracy }\end{array}$ & $\begin{array}{c}\text { TP, FP, } \\
\text { TN, FN, } \\
\text { sensitivity, } \\
\text { specificity, } \\
\text { PPV, NPV, } \\
\text { diagnostic } \\
\text { accuracy }\end{array}$ & $\begin{array}{c}\text { TP, FP, } \\
\text { TN, FN, } \\
\text { sensitivity, } \\
\text { specificity, } \\
\text { PPV, NPV, } \\
\text { diagnostic } \\
\text { accuracy }\end{array}$ & $\begin{array}{c}\text { TP, FP, } \\
\text { TN, FN, } \\
\text { sensitivity, } \\
\text { specificity, } \\
\text { diagnostic } \\
\text { accuracy }\end{array}$ & $\begin{array}{c}\text { TP, FP, } \\
\text { TN, FN, } \\
\text { sensitivity, } \\
\text { specificity, } \\
\text { PPV, NPV, } \\
\text { diagnostic } \\
\text { accuracy }\end{array}$ & $\begin{array}{c}\text { TP, FP, } \\
\text { TN, FN, } \\
\text { sensitivity, } \\
\text { specificity, } \\
\text { PPV, NPV, } \\
\text { diagnostic } \\
\text { accuracy }\end{array}$ \\
\hline Accuracy of results & $\mathrm{Y}$ & $\mathrm{Y}$ & $\mathrm{Y}$ & $\mathrm{Y}$ & $\mathrm{Y}$ & $\mathrm{Y}$ & $\mathrm{Y}$ & $\mathrm{Y}$ & $\mathrm{Y}$ & $\mathrm{Y}$ \\
\hline
\end{tabular}

Y: Yes; N: no; -: the assessor could not finalize a decision either way; CASP: Critical Appraisal Skills Programme; TP: true positive; FP: false positive; TN: true negative, FN: false negative; PPV: positive predictive value; NPV: negative predictive value.

SNB and ALND in those with a negative result (28). A positive axilla on PET/CT is considered a good indication of ALN involvement (31), due to the relatively high specificity.

Most studies in this review have only reported diagnostic performance when using only qualitative visual analysis of PET/CT scans of the axilla $(7-9,16,19-21)$. Kitajima et al. (3) have found that diagnostic performance was not significantly different between visual assessment and SUVmax analysis. When applying ${ }^{18}$ FDG PET/CT for the use of ALN staging, both visual and semi-qualitative analysis should be used (16).

Zhang et al. (13) have also found that when SUVmax is used separately, pooled sensitivity can increase slightly and specificity decreases significantly $(p<0.05)(13)$, however, similar to this review only a small number of papers could be included in their analysis. Higher ALN SUVmax can decrease false positive rates (16) as seen in a study by Ueda et al.'s (1), whereas SUVmax cut off points increase past 1.8, with specificity at $100 \%$ but sensitivity decreased to $36 \%$. Kim et al. (29) have suggested that lowering the SUVmax threshold to less than 1.05 can lead to sensitivity being $100 \%$, hence omitting all FNs. Potentially, false positive rates could be reduced by using SUVmax analysis (28). Visual analysis has been shown to be accurate and suggested as being easier to implement in practise (24).
Generally, it has been found that ${ }^{18} \mathrm{FDG}$ PET/CT has a moderate sensitivity and a high specificity for ALN metastasis (10), and we found pooled diagnostic accuracy to be $77.3 \%$.

The current literature, including studies in this review, is equivocal regarding the utility of ${ }^{18} \mathrm{FDG} \mathrm{PET} / \mathrm{CT}$. ${ }^{18} \mathrm{FDG}$ $\mathrm{PET} / \mathrm{CT}$ could be used to determine whether patients require SNB or ALND $(10,18)$, thereby avoiding a potentially unnecessary invasive procedure $(10,14)$. Some authors suggest that when ${ }^{18} \mathrm{FDG}$ PET/CT is positive patients could be offered ALND immediately $(8,28,32)$, whereas a negative finding on ${ }^{18} \mathrm{FDG} \mathrm{PET} / \mathrm{CT}$ indicates the continued need of SNB. This is due to the high FN rate observed (8). The FNR of $48 \%$ for PET/CT (sensitivity=52\%) compares with $10 \%$ for SNB (33). Therefore, we could not rely on a negative PET scan to avoid axillary surgery.

In contrast, other authors do not believe PET/CT should be routinely used (17) and could not replace current approaches for axillary staging (19), especially in view of its poor lesion-by-lesion-based sensitivity (19). Most studies we analysed did not directly compare PET/CT scan results with histological findings in a node-to-node manner, thereby limiting the conclusions that could have been drawn (13).

Some authors have suggested that PET/CT scans of the axilla could assist in therapeutic decision-making by revising the disease stage (34). 
Table III. A summary of diagnostic accuracy ${ }^{18}$ FDG-PET/CT scans for ALN metastasis when using visual, qualitative assessment. Where values or 95\% confidence intervals were not provided the authors calculated these.

\begin{tabular}{|c|c|c|c|c|c|c|c|c|c|}
\hline Study & $\mathrm{TP}$ & FP & $\mathrm{TN}$ & FN & $\begin{array}{c}\text { Sensitivity (\%) } \\
(95 \% \mathrm{CI})\end{array}$ & $\begin{array}{c}\text { Specificity (\%) } \\
(95 \% \mathrm{CI})\end{array}$ & $\begin{array}{l}\text { PPV (\%) } \\
(95 \% \mathrm{CI})\end{array}$ & $\begin{array}{l}\text { NPV (\%) } \\
(95 \% \mathrm{CI})\end{array}$ & $\begin{array}{c}\text { Accuracy (\%) } \\
(95 \% \mathrm{CI})\end{array}$ \\
\hline $\begin{array}{l}\text { Heusner } \text { et al. } \\
2009 \text { (19) }\end{array}$ & 14 & 3 & 34 & 10 & $\begin{array}{c}58 \\
(36.6-77.9)\end{array}$ & $\begin{array}{c}92 \\
(78.1-98.3)\end{array}$ & $\begin{array}{c}82 \\
(60.0-93.6)\end{array}$ & $\begin{array}{c}77 \\
(67.7-84.6)\end{array}$ & $\begin{array}{c}79 \\
(66.3-88.1)\end{array}$ \\
\hline $\begin{array}{l}\text { Jeong et al. } \\
2014 \text { (20) }\end{array}$ & 10 & 17 & 113 & 38 & $\begin{array}{c}20.8 \\
(10.5-35.0)\end{array}$ & $\begin{array}{c}86.9 \\
(79.9-92.2)\end{array}$ & $\begin{array}{c}37 \\
(22.5-54.4)\end{array}$ & $\begin{array}{c}74.8 \\
(71.7-77.7)\end{array}$ & $\begin{array}{c}69.1 \\
(61.8-75.80)\end{array}$ \\
\hline $\begin{array}{l}\text { Kong et al. } \\
2010 \text { (21) }\end{array}$ & 28 & 8 & 95 & 12 & $\begin{array}{c}70 \\
(53.5-83.4)\end{array}$ & $\begin{array}{c}92.2 \\
(85.3-96.6)\end{array}$ & $\begin{array}{c}77.8 \\
(63.6-87.5)\end{array}$ & $\begin{array}{c}88.8 \\
(83 .-92.7)\end{array}$ & $\begin{array}{c}86.0 \\
(79.2-91.2)\end{array}$ \\
\hline $\begin{array}{l}\text { Kutlutürk et al. } \\
2019 \text { (15) }\end{array}$ & 119 & 15 & 53 & 45 & $\begin{array}{c}72.6 \\
(65.1-79.2)\end{array}$ & $\begin{array}{c}77.9 \\
(66.2-87.1)\end{array}$ & $\begin{array}{c}88.8 \\
(83.4-92.6)\end{array}$ & $\begin{array}{c}54.1 \\
(47.1-60.9)\end{array}$ & $\begin{array}{c}74.1 \\
(68.0-79.6)\end{array}$ \\
\hline $\begin{array}{l}\text { Machida et al. } \\
2019 \text { (9) }\end{array}$ & 10 & 5 & 168 & 44 & $\begin{array}{c}18.5 \\
(9.3-31.4)\end{array}$ & $\begin{array}{c}97.1 \\
(93.4-99.1)\end{array}$ & $\begin{array}{c}66.7 \\
(41.7-84.8)\end{array}$ & $\begin{array}{c}79.2 \\
(77.0-81.3)\end{array}$ & $\begin{array}{c}78.4 \\
(72.5-83.6)\end{array}$ \\
\hline $\begin{array}{l}\text { Mori et al. } \\
2019 \text { (22) }\end{array}$ & 11 & 15 & 54 & 2 & $\begin{array}{c}85 \\
(54.6-98.1)\end{array}$ & $\begin{array}{c}78 \\
(66.7-87.3)\end{array}$ & $\begin{array}{c}42 \\
(30.7-54.8)\end{array}$ & $\begin{array}{c}96 \\
(88.2-99.0)\end{array}$ & $\begin{array}{c}79 \\
(68.9-87.4)\end{array}$ \\
\hline $\begin{array}{l}\text { Park et al. } \\
2017 \text { (7) }\end{array}$ & 17 & 6 & 105 & 16 & $\begin{array}{c}51.5 \\
(33.5-69.2)\end{array}$ & $\begin{array}{c}94.6 \\
(88.6-98.0)\end{array}$ & $\begin{array}{c}73.9 \\
(54.9-86.8)\end{array}$ & $\begin{array}{c}86.8 \\
(82.2-90.3)\end{array}$ & $\begin{array}{c}84.7 \\
(77.8-90.2)\end{array}$ \\
\hline $\begin{array}{l}\text { Ueda et al. } \\
2008 \text { (1) }\end{array}$ & 34 & 6 & 118 & 25 & $\begin{array}{c}57.6 \\
(44.1-70.4)\end{array}$ & $\begin{array}{c}95.2 \\
(89.8-98.2)\end{array}$ & $\begin{array}{c}85 \\
(71.6-92.7)\end{array}$ & $\begin{array}{c}82.5 \\
(77.8-86.4)\end{array}$ & $\begin{array}{c}83.1 \\
(76.8-88.2)\end{array}$ \\
\hline $\begin{array}{l}\text { Veronesi et al. } \\
2007 \text { (8) }\end{array}$ & 38 & 5 & 128 & 65 & $\begin{array}{c}37 \\
(27.6-47.0)\end{array}$ & $\begin{array}{c}96 \\
(91.4-98.8)\end{array}$ & $\begin{array}{c}88 \\
(75.6-94.9)\end{array}$ & $\begin{array}{c}66 \\
(62.9-69.6)\end{array}$ & $\begin{array}{c}70 \\
(64.1-76.1)\end{array}$ \\
\hline
\end{tabular}

TP: True positive; FP: false positive; TN: true negative, FN: false negative; CI: confidence interval; PPV: positive predictive value; NPV: negative predictive value.

Table IV. A summary of diagnostic accuracy ${ }^{18}$ FDG-PET/CT scans for ALN metastasis when using SUVmax assessment. Where values or 95\% confidence intervals were not provided the authors calculated these.

\begin{tabular}{|c|c|c|c|c|c|c|c|c|c|c|}
\hline Study & $\begin{array}{l}\text { SUVmax } \\
\text { Cut off }\end{array}$ & $\mathrm{TP}$ & FP & $\mathrm{TN}$ & $\mathrm{FN}$ & $\begin{array}{l}\text { Sensitivity (\%) } \\
(95 \% \mathrm{CI})\end{array}$ & $\begin{array}{l}\text { Specificity (\%) } \\
(95 \% \mathrm{CI})\end{array}$ & $\begin{array}{l}\text { PPV (\%) } \\
(95 \% \mathrm{CI})\end{array}$ & $\begin{array}{l}\text { NPV (\%) } \\
(95 \% \mathrm{CI})\end{array}$ & $\begin{array}{c}\text { Accuracy }(\%) \\
(95 \% \mathrm{CI})\end{array}$ \\
\hline $\begin{array}{l}\text { Chae et al. } \\
2008 \text { (2) }\end{array}$ & Unknown & 16 & 12 & 63 & 17 & $\begin{array}{c}48.5 \\
(30.8-66.5)\end{array}$ & $\begin{array}{c}84 \\
(73.7-91.5)\end{array}$ & $\begin{array}{c}\text { Unknown } \\
57.1 \\
(41.6-71.4)\end{array}$ & $\begin{array}{c}\text { Unknown } \\
78.8 \\
(72.4-84.0)\end{array}$ & $\begin{array}{c}73.2 \\
(63.8-81.2)\end{array}$ \\
\hline $\begin{array}{l}\text { Mori et al. } \\
2019 \text { (22) }\end{array}$ & 1.1 & & & & & 69 & 99 & 90 & 94 & 94 \\
\hline $\begin{array}{l}\text { Ueda et al. } \\
2008 \text { (1) }\end{array}$ & 0.8 & 30 & 6 & 118 & 29 & $\begin{array}{c}50.8 \\
(37.5-64.1)\end{array}$ & $\begin{array}{c}95.2 \\
(89.8-98.2)\end{array}$ & $\begin{array}{c}83.3 \\
(68.8-91.9)\end{array}$ & $\begin{array}{c}80.3 \\
(75.8-84.1)\end{array}$ & $\begin{array}{c}80.9 \\
(74.4-86.3)\end{array}$ \\
\hline $\begin{array}{l}\text { Ueda et al. } \\
2008 \text { (1) }\end{array}$ & 1.3 & 24 & 2 & 122 & 35 & $\begin{array}{c}40.7 \\
(28.1-54.3)\end{array}$ & $\begin{array}{c}98.4 \\
(94.3-99.8)\end{array}$ & $\begin{array}{c}92.3 \\
(74.6-98.0)\end{array}$ & $\begin{array}{c}77.7 \\
(73.8-81.2)\end{array}$ & $\begin{array}{c}79.8 \\
(73.23-85.4)\end{array}$ \\
\hline $\begin{array}{l}\text { Ueda et al. } \\
2008 \text { (1) }\end{array}$ & 1.5 & 21 & 1 & 123 & 38 & $\begin{array}{c}35.6 \\
(23.6-49.1)\end{array}$ & $\begin{array}{c}99.2 \\
(95.6-100)\end{array}$ & $\begin{array}{c}99.5 \\
(74.3-99.4)\end{array}$ & $\begin{array}{c}76.4 \\
(72.78-79.7)\end{array}$ & $\begin{array}{c}78.7 \\
(72.0-84.4)\end{array}$ \\
\hline $\begin{array}{l}\text { Ueda et al. } \\
2008 \text { (1) }\end{array}$ & 1.8 & 21 & 0 & 124 & 38 & $\begin{array}{c}35.6 \\
(23.6-49.1)\end{array}$ & $\begin{array}{c}100 \\
(97.1-100)\end{array}$ & 100 & $\begin{array}{c}76.5 \\
(727.0-79.8)\end{array}$ & $\begin{array}{c}79.2 \\
(72.6-84.9)\end{array}$ \\
\hline $\begin{array}{l}\text { Ueda et al. } \\
2008 \text { (1) }\end{array}$ & 2 & 20 & 0 & 124 & 39 & $\begin{array}{c}33.9 \\
(22.1-47.39)\end{array}$ & $\begin{array}{c}100 \\
(97.1-100)\end{array}$ & 100 & $\begin{array}{c}76.1 \\
(72.6-79.2)\end{array}$ & $\begin{array}{c}78.7 \\
(72.0-84.4)\end{array}$ \\
\hline $\begin{array}{l}\text { Ueda et al. } \\
2008 \text { (1) }\end{array}$ & 3 & 16 & 0 & 124 & 43 & $\begin{array}{c}27.1 \\
(16.4-40.3)\end{array}$ & $\begin{array}{c}100 \\
(97.1-100)\end{array}$ & 100 & $\begin{array}{c}74.3 \\
(71.2-77.1)\end{array}$ & $\begin{array}{c}76.5 \\
(69.7-82.4)\end{array}$ \\
\hline
\end{tabular}

SUVmax: Maximum standardized uptake value; TP: true positive; FP: false positive; TN: true negative, FN: false negative; CI: confidence interval; PPV: positive predictive value; NPV: negative predictive value.

The limitations of this study are that it included mostly retrospective papers, hence a selection bias might have occurred when selecting patients. In addition, as most papers did not include a lesion-by-lesion analysis, it is more difficult to ascertain the true diagnostic accuracy of this staging method. Lastly, we did not perform a heterogeneity test; however, we did assess the quality of the papers included through the use of a critical analysis tool.

Further research is required to find a consensus on the use of ${ }^{18}$ FDG PET/CT scans in axillary staging. Specifically, 
there is a need for prospective studies assessing whether it could help in deciding whether a patient should have an SNB or proceed directly to ALND, thus, avoiding unnecessary invasive procedures.

In addition, further research is necessary to ascertain whether the qualitive visual method is comparable to the semi-qualitative method using SUVmax. Furthermore, clinically useful SUVmax thresholds need to be delineated. Finally, it needs to be clarified whether primary tumour size is a determinant of the efficacy of ${ }^{18}$ FDG-PET/CT in axillary staging.

In conclusion, it was observed that overall ${ }^{18}$ FDGPET/CT offers low sensitivity but high specificity for axillary lymph node metastatic disease. Visual analysis proved to be a fair method when assessing PET/CT scans, however, it cannot be used as the sole method to evaluate the ALN status nor can it replace the current staging procedures. ${ }^{18}$ FDG-PET/CT could potentially be used to direct whether a patient requires SNB prior to ALND, however, additional prospective research should be conducted before guidelines for a conclusive recommendation could be made.

\section{Conflicts of Interest}

The Authors do not have any conflicts of interest to declare in relation to this article.

\section{Authors' Contributions}

KM supervised and initialised the project. JK drafted the initial manuscript. UW performed the literature search and produced the final draft of the manuscript.

\section{Acknowledgements}

This work was made possible by support from the Breast Cancer Hope Foundation, UK.

\section{References}

1 Ueda S, Tsuda H, Asakawa H, Omata J, Fukatsu K, Kondo N, Kondo T, Hama Y, Tamura K, Ishida J, Abe Y and Mochizuki H: Utility of 18f-fluoro-deoxyglucose emission tomography/ computed tomography fusion imaging $\left({ }^{18} \mathrm{f}-\mathrm{fdg}\right.$ pet/ct) in combination with ultrasonography for axillary staging in primary breast cancer. BMC Cancer 8: 165, 2008. PMID: 18541009. DOI: $10.1186 / 1471-2407-8-165$

2 Chae BJ, Bae JS, Kang BJ, Kim SH, Jung SS and Song BJ: Positron emission tomography-computed tomography in the detection of axillary lymph node metastasis in patients with early stage breast cancer. Jpn J Clin Oncol 39(5): 284-289, 2009. PMID: 19318373. DOI: 10.1093/jjco/hyp019

3 Kitajima K, Fukushima K, Miyoshi Y, Katsuura T, Igarashi Y, Kawanaka Y, Mouri M, Maruyama K, Yamano T, Doi H, Yamakado K, Hirota S and Hirota S: Diagnostic and prognostic value of (18)f-fdg pet/ct for axillary lymph node staging in patients with breast cancer. Jpn J Radiol 34(3): 220-228, 2016. PMID: 26715510. DOI: $10.1007 / \mathrm{s} 11604-015-0515-1$
4 NICE. Early and locally advanced breast cancer. National Institute for Health \& Clinical Excellance; 18 July 2018. Report No.: NG101.

5 Gera R, Kasem A and Mokbel K: Can complete axillary node dissection be safely omitted in patients with early breast cancer when the sentinel node biopsy is positive for malignancy? An update for clinical practice. In Vivo 32(6): 1301-1307, 2018. PMID: 30348682. DOI: 10.21873/invivo.11380

6 Blodgett TM, Meltzer CC and Townsend DW: Pet/ct: Form and function. Radiology 242(2): 360-385, 2007. PMID: 17255408. DOI: $10.1148 /$ radiol 2422051113

7 Park HL, Yoo IR, O JH, Kim H, Kim SH and Kang BJ: Clinical utility of ${ }^{18} \mathrm{f}$-fdg pet/ct in low ${ }^{18} \mathrm{f}$-fdg-avidity breast cancer subtypes: Comparison with breast us and mri. Nucl Med Commun 39(1): 35-43, 2018. PMID: 28984814. DOI: 10.1097/MNM.0000000000000768

8 Veronesi U, De Cicco C, Galimberti VE, Fernandez JR, Rotmensz N, Viale G, Spano G, Luini A, Intra M, Veronesi P, Berrettini A and Paganelli G: A comparative study on the value of fdg-pet and sentinel node biopsy to identify occult axillary metastases. Ann Oncol 18(3): 473-478, 2007. PMID: 17164229. DOI: $10.1093 /$ annonc/mdl425

9 Machida Y, Kubota K, Katayama T, Toriihara A and Shibuya H: Diagnostic performance of fluorodeoxyglucose-positron emission tomography/computed tomography combined with ultrasonography-guided fine needle aspiration cytology for identifying axillary lymph node status in patients with breast cancer. Eur J Surg Oncol 39(1): 26-30, 2013. PMID: 23122913. DOI: $10.1016 /$ j.ejso.2012.10.012

10 Ozkan EE, Sengul SS, Erdogan M, Gurdal O and Eroglu HE: ${ }^{18} \mathrm{f}-$ fluorodeoxyglucose pet/computed tomography in locoregional staging and assessment of biological and clinical aggressiveness of breast cancer subtypes. Nucl Med Commun 40(10): 1043-1050, 2019. PMID: 31425343 . DOI: 10.1097/MNM.0000000000001073

11 Grueneisen J, Nagarajah J, Buchbender C, Hoffmann O, Schaarschmidt BM, Poeppel T, Forsting M, Quick HH, Umutlu $\mathrm{L}$ and Kinner S: Positron emission tomography/magnetic resonance imaging for local tumor staging in patients with primary breast cancer: A comparison with positron emission tomography/computed tomography and magnetic resonance imaging. Invest Radiol 50(8): 505-513, 2015. PMID: 26115367. DOI: 10.1097/RLI.0000000000000197

12 van Uden DJP, Prins MW, Siesling S, de Wilt JHW, BlankenPeeters $\mathrm{C}$ and Aarntzen E: $\left[{ }^{18} \mathrm{f}\right] \mathrm{fdg}$ pet/ct in the staging of inflammatory breast cancer: A systematic review. Crit Rev Oncol Hematol 151: 102943, 2020. PMID: 32416347. DOI: 10.1016/j.critrevonc.2020.102943

13 Zhang X, Liu Y, Luo $\mathrm{H}$ and Zhang J: Pet/ct and mri for identifying axillary lymph node metastases in breast cancer patients: Systematic review and meta-analysis. J Magn Reson Imaging, 2020. PMID: 32567090. DOI: 10.1002/jmri.27246

14 Robertson IJ, Hand F and Kell MR: Fdg-pet/ct in the staging of local/regional metastases in breast cancer. Breast 20(6): 491-494, 2011. PMID: 21807517. DOI: 10.1016/j.breast.2011.07.002

15 Sohn YM, Hong IK and Han K: Role of [ $\left.{ }^{18} \mathrm{f}\right]$ fluorodeoxyglucose positron emission tomography-computed tomography, sonography, and sonographically guided fine-needle aspiration biopsy in the diagnosis of axillary lymph nodes in patients with breast cancer: Comparison of diagnostic performance. J Ultrasound Med 33(6): 1013-1021, 2014. PMID: 24866608. DOI: 10.7863/ultra.33.6.1013 
16 Kutluturk K, Simsek A, Comak A, Gonultas F, Unal B and Kekilli E: Factors affecting the accuracy of (18)f-fdg pet/ct in evaluating axillary metastases in invasive breast cancer. Niger J Clin Pract 22(1): 63-68, 2019. PMID: 30666022. DOI: 10.4103/njcp.njcp_198_18

17 Monzawa S, Adachi S, Suzuki K, Hirokaga K, Takao S, Sakuma $\mathrm{T}$ and Hanioka K: Diagnostic performance of fluorodeoxyglucose-positron emission tomography/computed tomography of breast cancer in detecting axillary lymph node metastasis: Comparison with ultrasonography and contrast-enhanced ct. Ann Nucl Med 23(10): 855-861, 2009. PMID: 19876704. DOI: 10.1007/s12149-009-0314-9

18 Fuster D, Duch J, Paredes P, Velasco M, Munoz M, Santamaria G, Fontanillas M and Pons F: Preoperative staging of large primary breast cancer with [ ${ }^{18}$ f]fluorodeoxyglucose positron emission tomography/computed tomography compared with conventional imaging procedures. J Clin Oncol 26(29): 47464751, 2008. PMID: 18695254. DOI: 10.1200/JCO.2008.17.1496

19 Heusner TA, Kuemmel S, Hahn S, Koeninger A, Otterbach F, Hamami ME, Kimmig KR, Forsting M, Bockisch A, Antoch G and Stahl A: Diagnostic value of full-dose fdg pet/ct for axillary lymph node staging in breast cancer patients. Eur J Nucl Med Mol Imaging 36(10): 1543-1550, 2009. PMID: 19415270. DOI: 10.1007/s00259-009-1145-6

20 Jeong YJ, Kang DY, Yoon HJ and Son HJ: Additional value of f-18 fdg pet/ct for initial staging in breast cancer with clinically negative axillary nodes. Breast Cancer Res Treat 145(1): 137142, 2014. PMID: 24682676. DOI: 10.1007/s10549-014-2924-8

21 Kong EJ, Chun KA, Cho IH and Lee SJ: (18)f-fdg pet/ct with contrast enhancement for evaluation of axillary lymph node involvement in t1 breast cancer. Nucl Med Mol Imaging 44(3): 170-176, 2010. PMID: 24899946. DOI: 10.1007/s13139-0100035-y

22 Mori M, Fujioka T, Katsuta L, Tsuchiya J, Kubota K, Kasahara M, Oda G, Nakagawa T, Onishi I and Tateishi U: Diagnostic performance of time-of-flight pet/ct for evaluating nodal metastasis of the axilla in breast cancer. Nucl Med Commun 40(9): 958-964, 2019. PMID: 31365505. DOI: 10.1097/MNM.00 00000000001057

23 Sasada S, Kimura Y, Emi A, Masumoto N, Kadoya T, Arihiro K and Okada M: Tumor-infiltrating lymphocyte score based on fdg pet/ct for predicting the effect of neoadjuvant chemotherapy in breast cancer. Anticancer Res 40(6): 3395-3400, 2020. PMID: 32487636. DOI: 10.21873/anticanres.14323

24 Collaborators GBDCoD: Global, regional, and national age-sex specific mortality for 264 causes of death, 1980-2016: A systematic analysis for the global burden of disease study 2016. Lancet 390(10100): 1151-1210, 2017. PMID: 28919116. DOI: 10.1016/S0140-6736(17)32152-9

25 Vandenberghe S, Mikhaylova E, D'Hoe E, Mollet P and Karp JS: Recent developments in time-of-flight pet. EJNMMI Phys 3(1): 3, 2016. PMID: 26879863. DOI: 10.1186/s40658-016-0138-3
26 Koolen BB, van der Leij F, Vogel WV, Rutgers EJ, Vrancken Peeters MJ, Elkhuizen PH and Valdes Olmos RA: Accuracy of $18 \mathrm{f}$-fdg pet/ct for primary tumor visualization and staging in $\mathrm{t} 1$ breast cancer. Acta Oncol 53(1): 50-57, 2014. PMID: 23672678. DOI: $10.3109 / 0284186 X .2013 .783714$

27 Riegger C, Herrmann J, Nagarajah J, Hecktor J, Kuemmel S, Otterbach F, Hahn S, Bockisch A, Lauenstein T, Antoch G and Heusner TA: Whole-body fdg pet/ct is more accurate than conventional imaging for staging primary breast cancer patients. Eur J Nucl Med Mol Imaging 39(5): 852-863, 2012. PMID: 22392069. DOI: $10.1007 / \mathrm{s} 00259-012-2077-0$

28 Wahl RL, Siegel BA, Coleman RE, Gatsonis CG and Group PETS: Prospective multicenter study of axillary nodal staging by positron emission tomography in breast cancer: A report of the staging breast cancer with pet study group. J Clin Oncol 22(2): 277-285, 2004. PMID: 14722036 . DOI: $10.1200 / J C O .2004 .04 .148$

29 Kim J, Lee J, Chang E, Kim S, Suh K, Sul J, Song I, Kim Y and Lee C: Selective sentinel node plus additional non-sentinel node biopsy based on an fdg-pet/ct scan in early breast cancer patients: Single institutional experience. World J Surg 33(5): 943-949, 2009. PMID: 19259728. DOI: 10.1007/s00268-009-9955-z

30 Salhab M, Patani N and Mokbel K: Sentinel lymph node micrometastasis in human breast cancer: An update. Surg Oncol 20(4): e195-206, 2011. PMID: 21788132. DOI: 10.1016/ j.suronc.2011.06.006

31 Choi YJ, Shin YD, Kang YH, Lee MS, Lee MK, Cho BS, Kang YJ and Park JS: The effects of preoperative (18)f-fdg pet/ct in breast cancer patients in comparison to the conventional imaging study. J Breast Cancer 15(4): 441-448, 2012. PMID: 23346174. DOI: $10.4048 /$ jbc.2012.15.4.441

32 Chandra P, Ravichander SK, Babu SM, Jain D and Nath S: Evaluation of diagnostic accuracy and impact of preoperative positron emission tomography/computed tomography in the management of early operable breast cancers. Indian J Nucl Med 35(1): 40-47, 2020. PMID: 31949368. DOI: 10.4103/ijnm. IJNM_140_19

33 Wazir U and Mokbel K: De-escalation of axillary surgery in the neoadjuvant chemotherapy (nact) setting for breast cancer: Is it oncologically safe? Anticancer Res 40(10): 5351-5354, 2020. PMID: 32988853. DOI: 10.21873/anticanres.14542

34 Garami Z, Hascsi Z, Varga J, Dinya T, Tanyi M, Garai I, Damjanovich L and Galuska L: The value of $18-\mathrm{fdg}$ pet/ct in early-stage breast cancer compared to traditional diagnostic modalities with an emphasis on changes in disease stage designation and treatment plan. Eur J Surg Oncol 38(1): 31-37, 2012. PMID: 21937190. DOI: 10.1016/j.ejso.2011.09.002

Received October 13, 2020

Revised November 4, 2020

Accepted November 6, 2020 